\title{
UNITARY MATRICES AS BOUNDARY VALUES OF ANALYTIC FUNCTIONS
}

\author{
O. S. ROTHAUS
}

Let $Z$ be a matricial variable, and $F(Z)$ an analytic matrix valued function. Under what circumstances is $F(Z)$ unitary whenever $Z$ is unitary? We can obtain a partial answer to this question in case $F(Z)$ is entire.

We consider the function $F(z U)$ of a single complex variable $z$, where $U$ is unitary. $F(z U)$ is a function of the type already considered by Potapov [2], and we can adapt his results to obtain our conclusions.

A matrix valued function $F(z)=\left[F_{i j}(z)\right]$ is simply an $n$ by $n$ square array of functions defined for all $z$ in a domain $D$, usually the unit disc. We will say that $F(z)$ has a property such as analyticity, boundedness, etc., if the property is true of all the component functions.

Now let $F(z)$ be a matrix valued function, analytic in the open unit $\operatorname{disc}|z|<1$ and continuous in the closed unit disc. We will suppose further that $F(z)$ is unitary when $|z|=1$. The collection $S$ of such functions forms a semigroup under (matrix) multiplication. Our first results will give the structure of this semigroup. It is convenient to set some notation:

(i) $\|F(z)\|$ is the determinant of $F(z)$.

(ii) $F^{T}(z)$ is the transpose of $F(z)$.

(iii) $F^{*}(z)$ is the conjugate transpose of $F(z)$.

(iv) $f_{\alpha}(z)=|\alpha| / \alpha \cdot(\alpha-z) /(1-\bar{\alpha} z), 0<|\alpha|<1$, and $f_{0}(z)=z$, called Blaschke factors.

(v) $F_{\alpha}(z)$ is the matrix

$$
\left[\begin{array}{cc}
f_{\alpha}(z) & 0 \\
0 & I_{n-1}
\end{array}\right]
$$

where $I_{n-1}$ is the $n-1$ dimensional identity matrix. (Note that $F_{\alpha}(z)$ is unitary for $|z|=1$.)

(vi) $U_{v}$ will always denote a unitary matrix.

We start with the following simple lemma, a variant of the Schwarz reflection principle.

Lemma 1. Let $F(z)$ be as described above. Suppose in addition that $\|F(z)\|$ has no zeroes in $|z|<1$. Then $F(z)$ is a constant (necessarily a unitary matrix).

Received by the editors December 7, 1965 and, in revised form, June 28, 1965. 
Since $\|F(z)\| \neq 0$ for $|z| \leqq 1, F^{-1}(z)$ is analytic in $|z|<1$ and continuous in $|z| \leqq 1$. On the unit circle $|z|=1$ we have

$$
F(z) F^{*}(z)=I
$$

which we write

$$
F(z) F^{*}(1 / \bar{z})=I
$$

or

$$
F(z)=\left(F^{*}(1 / \bar{z})\right)^{-1} \text { for }|z|=1 .
$$

But the expression of the right is analytic for $|z|>1$, and so gives an analytic continuation of $F(z)$ into the extended plane. Consequently, $F(z)$ is constant.

Returning to the general case, note that $\|F(z)\|$ is analytic in $|z|<1$, continuous in $|z| \leqq 1$ and of absolute value one on $|z|=1$. Consequently, $\|F(z)\|$ is a constant (of absolute value one) times a finite product of Blaschke factors,

$$
\|F(z)\|=e^{i \lambda} \prod_{v=1}^{k} f_{\alpha_{v}}(z) .
$$

We have $\left\|F\left(\alpha_{1}\right)\right\|=0$. Let $u$ be a row vector of length one in the nullspace of $F\left(\alpha_{1}\right)$ :

$$
u F\left(\alpha_{1}\right)=0 .
$$

We construct a unitary matrix $U_{1}$ whose first row is $u$, so that $U_{1} F\left(\alpha_{1}\right)$ has a first row identically zero. Thus $U_{1} F(z)$ has a first row vanishing at $z=\alpha_{1}$. Consequently, the function $G(z)=\left(F_{\alpha_{1}}(z)\right)^{-1} U_{1} F(z)$ is regular in $|z|<1$, and, as is immediately verified, belongs to $s$. $\|G(z)\|$ has one Blaschke factor less in its expansion; i.e.,

$$
\|G(z)\|=e^{i \lambda_{1}} \prod_{v=2}^{k} f_{\alpha_{v}}(z)
$$

After a finite number of such reductions, we arrive at a function to which the result of Lemma 1 is applicable. We have proved:

THEOREM 1. The semigroup $\delta$ is generated by unitary matrices and matrices $F_{\alpha}(z)(0 \leqq|\alpha|<1)$.

If we had assumed at the start that $F(z)$ was an entire function, then we would have $\|F(z)\|=e^{i \lambda} z^{k}$. Consequently:

COROLlary 1. The sub-semigroup of $S$ consisting of entire functions is generated by the unitary matrices and the single matrix $F_{0}(z)$. Further- 
more, if $F(z)$ is an entire function in $S$, then $\|F(z)\|=e^{i \lambda} z^{k}$, and the entries in $F(z)$ are all polynomials of degree at most $k$.

Potapov has many interesting results on the uniform closure of the semigroup $s$.

We return now to the question in the initial paragraph of this paper. We have that

$$
\|F(z U)\|=c(U) z^{K(U)}
$$

where, in fact, since the unitary group is connected, $K(U)$ must be constant $K$. Now from Corollary 1 , we know that the entries in $F(z U)$ are all polynomials of degree at most $K$. Hence in the expansion of $F(Z)$ in a power series, the homogeneous terms of weight greater than $K$ must vanish on the unitary group. This is known to insure their vanishing identically. Thus we have:

Theorem 2. Under the hypothesis described above, the entries in $F(Z)$ are polynomials in the entries of $Z$.

Somewhat more can be proved easily. Our argument above will show that $\|F(Z)\|$ is a homogeneous polynomial of weight $K$. For $Z$ unitary we have:

$$
F(Z) F^{*}(Z)=I
$$

which we write:

$$
F(Z) F^{*}\left(Z^{*-1}\right)=I .
$$

Since $F^{*}\left(Z^{*-1}\right)$ is analytic where it is defined, the above equation is true for all $Z$. Put ad $Z=\|Z\| Z^{-1}$. ad $Z$ is a polynomial in $Z$. By taking determinants above we obtain:

$$
\|F(Z)\| \| F^{*}\left(\frac{1}{\left\|Z^{*}\right\|} \text { ad } Z^{*}\right) \|=I
$$

Using the homogeneity of $\|F(Z)\|$ we have:

$$
\|F(Z)\|\left\|F^{*}\left(\operatorname{ad} Z^{*}\right)\right\|=\|Z\| \text {. }
$$

Since $\|Z\|$ is irreducible, $\|F(Z)\|=c\|Z\| L$.

In the special case that the dimension of $Z$ is that of $F(Z)$, we note the following entire functions which are unitary when $Z$ is unitary:

(i) $Z$,

(ii) $Z^{T}$,

(iii) $\operatorname{ad} Z$,

(iv) ad $Z^{T}$, 


$$
\left(\begin{array}{cc}
\|Z\| & 0 \\
0 & I
\end{array}\right)
$$

It is possible that these together with the constant unitary matrices generate the whole class of such functions, but we have not been able to prove it.

\section{REFERENCES}

1. I. Priwalow, Randeigenschaften Analytisches Functionen, VEB Deutscher Verlag Wissenschaften, Berlin, 1956.

2. V. P. Potapov, The multiplicative structure of J-contractive matrix functions, Amer. Math. Soc. Transl. (2) 15 (1960), 131-243.

Institute for Defense Analyses, Princeton, New Jersey

\section{ON THE RADIUS OF UNIVALENCE OF CERTAIN ANALYTIC FUNCTIONS}

\section{A. E. LIVINGSTON}

Let $\mathfrak{C}$ denote the class of functions $f$ regular and univalent in $E=\{z|| z \mid<1\}$, which satisfy $f(0)=0$ and $f^{\prime}(0)=1$ and which are close-to-convex in $E$. Let $\mathcal{K}$ and $\mathcal{S}^{*}$ denote the subfamilies of $\mathcal{C}$, made up of functions which are convex and starlike in $E$, respectively. Recently, Libera [2] has shown that if $f$ is a member of $\Re$, $\mathcal{S}^{*}$ or $\mathfrak{e}$, then the function $F(z)=(2 / z) \int_{0}^{z} f(t) d t$ is also a member of $\Re, \varsigma^{*}$ or $\mathcal{C}$. It is the purpose of this paper to investigate the converse question. That is, if $F$ is in $\delta^{*}$, what is the radius of starlikeness of the function $f(z)=[1 / 2][z F(z)]^{\prime}$ ? Similar questions are answered under the assumption that $F$ is in $\nVdash$ or in $\mathcal{C}$. Robinson [5] has shown that if $F$ is only assumed to be univalent in $E$, then $f$ is starlike for $|z|<.38$. He pointed out that it is probable that $f$ is univalent for $|z|<(1 / 2)$. We obtain this result under the added assumption that $F$ is a member of $\Re, s^{*}$ or $\mathcal{C}$.

The method of proof used in Theorem 1 has recently been employed by MacGregor [4].

Presented to the Society, December 6, 1965; received by the editors April 28, 1965. 